\title{
Posterior Alpha Power During Working Memory Maintenance in Alcohol Use Disorder
}

\author{
Natalie Eve Rilo \\ University of Florida \\ Faculty Mentor: Sara Jo Nixon, Department of Psychiatry
}

\begin{abstract}
Individuals with alcohol use disorder (AUD) display modest neurobehavioral deficits on a range of cognitive tasks including those measuring working memory (WM). WM tasks involve multiple phases. A critical and understudied component is the maintenance phase, during which information must retained for later retrieval. To better understand neural activity during the maintenance phase, alpha power, an electrophysiologic index of inhibition was investigated. Healthy controls (HC) and newly abstinent individuals with AUD completed a directed attend/ignore WM task that used emotional faces. Spectral alpha power was measured from posterior electrode sites during a 9 second WM maintenance period. A mixed linear model compared posterior alpha power with group (HC vs. AUD) and sex (male vs. female) as between group factors within emotion. Main effects for group $(\mathrm{p}=.01)$ and $\operatorname{sex}(\mathrm{p}=.003)$ were significant. HC displayed a higher alpha power compared to that of individuals with AUD. Males also presented with higher alpha power compared to women. There was no significant interaction between group and sex. An exploratory analysis to determine a relationship between alpha power and performance on the task was conducted and a differential relationship between groups $(\mathrm{p}<.001)$ was found. There was no significant relationship between performance and spectral power for HC, but a negative relationship was found for individuals with AUD. Higher alpha power was associated with decreased accuracy $(p=.05)$. The decreased alpha power seen in individuals with AUD may initially be considered a decrement. However, the inverse relationship with performance suggests it may represent behavioral or compensatory strategies utilized by those with AUD. This remains a question intended for future investigation.

Keywords: alcohol use disorder, working memory, alpha power
\end{abstract}

\section{Introduction}

\section{Working Memory}

Working memory (WM) is the process by which information is temporarily stored and integrated for later manipulation and use (Baddeley, 1998; Baddeley, 2003; Corbetta \& Shulman, 2002). Once thought to be a part of short-term memory processing, WM is now considered to be an independent construct due to its unique simultaneous storage and processing of information (Baddeley, 2003). 
WM involves three main phases - encoding, maintaining and retrieving information. During encoding, one must attend to a stimulus whilst filtering out other irrelevant information in the environment. Once an item has been successfully encoded, the maintenance phase occurs. Maintenance is a brief period in which the encoded information is held for later manipulation and retrieval. This step protects the integrity of a stimulus in order to access the information at a later point in time. Finally, retrieval occurs through one of two steps: recognition or recall. Recognition refers to the ability to identify some piece of information as familiar when given a cue, while recall requires accessing information from memory without a cue. (Baddeley, 1998; Baddeley, 2003; Corbetta and Shulman, 2002). Recognition is a simpler process as it only asks whether a stimulus has previously been seen (binary yes/no) whereas recall requires full recovery and replication of the stimulus.

The more one can attribute meaning to a stimulus, the deeper it is processed, resulting in greater probability that one will encode it (Martin, 1968). For example, faces particularly those expressing salient emotion (Seidel et al, 2010) are more successfully retrieved than unexpressive faces or non-salient stimuli like landscapes.

\section{Alpha Power Density}

While the three steps of WM each uniquely contribute to an individual's WM performance, it is not feasible to examine how each stage independently functions by analyzing behavioral measures. To better answer these questions, researchers can use neuroimaging techniques to capture neural activity during each stage. Due to the speed with which these processes can occur a method such as electroencephalography (EEG), known for its precise temporal resolution, is optimal in decomposing the stages of WM.

Spectral power analyses, collected via EEG, measure the rate at which neurons fire, creating waveforms of varying frequencies (e.g. delta, theta, alpha, beta; Stoica \& Moses, 2005; Cohen 2017). By examining a waveform's power, or the strength and pattern of its amplitude (Geering, Achermann, Eggimann, \& Borbely 1993), widespread neural activity across the scalp can be evaluated.

While multiple waveforms have been identified, this project will be limited to alpha waves. The alpha frequency ranges from $7-12 \mathrm{~Hz}$ and is predominantly seen in the parietal and posterior portions of the scalp. It is heightened when attention is focused inward (e.g. at rest, during introspection). The maintenance phase of WM processes requires inward attention while 
blocking out external distractors. For this reason, alpha power is a commonly used as a neural correlate of WM maintenance.

It is not clear whether maintenance of emotional stimuli would require less alpha power due to the increased attention allocation given during encoding (Gonzalez-Garrido et al. 2015; Lee \& Cho, 2019); or whether greater alpha power would be prioritized due to the emotional salience (Mather \& Sutherland, 2011). The current project will examine alpha power during mental rehearsal of emotional faces.

\section{Alcohol Use Disorder}

Alcohol use disorder (AUD) is a chronic psychophysiological disease characterized by an impaired ability to stop or control alcohol use despite consequences (American Psychiatric Association, 2013). Although only a small percentage of people who regularly use alcohol are diagnosed with AUD, once it manifests, the neurobehavioral changes can be distinct and profound (Oscar-Berman \& Marinkovic, 2007; Hoffman, Lewis, Nixon 2019; Lewis, Price, Garcia, Nixon 2019; Nixon \& Glenn 2002; Beatty, Tivis, Stott, Nixon, Parsons 2000).

However, some groups have noted no performance deficits on cognitive tasks (Chanraud et al., 2007), deficits on some cognitive domains but not others (Oscar-Berman et al., 2014;

Tedstone \& Coyle, 2004), or neural alterations in the absence of cognitive deficits (Chanraud et al., 2012; Mueller-Oehring et al., 2014). In the case of the latter, bidirectional changes in neural activity have been seen and are dependent on task and brain region (Bernardin, Maheut-Bosser \& Paille 2014; Gould 2010). The recently expanding literature on memory for affective stimuli has pointed to socio-affective deficits in individuals with AUD. As working memory is consistently noted as an alcohol sensitive neurocognitive component, the inclusion of affective stimuli may further enhance or impair cognition and its neural correlates.

While the aforementioned patterns of the alpha frequency (i.e. heightened when attention is focused inward) exist in AUD at rest, disruptions in these wave forms are still apparent (Porjesz $\&$ Begleiter 2003). Heavy drinkers display less power in the alpha frequency during rest and maintenance states compared to that of healthy controls (HC) (deBruin 2006). While this speaks to individuals who partake in chronic alcohol use, it does not address the question of individuals with a clinical AUD diagnosis. 


\section{Current Study}

The current project replicates previously used methods (Boissoneault 2016) to extract PAP during the maintenance phase of a WM task to investigate differences between men and women with and without AUD. Previous reports on PAP during WM maintenance are mixed, some showing PAP elevations are protective, other showing decreases associated with better performance (deBruin 2006, Porjesz \& Begleiter 2003). For this reason, the current aims were tested with two-tailed tests. We expect H1: for individuals with AUD to have different PAP than controls during WM maintenance. We also explore E1: whether presumed differences in PAP relate to performance on the task (previously reported in Lewis et al., under review in revision).

\section{Materials and Methods}

\section{Recruitment and Participants}

Healthy control participants ( $\mathrm{N}=61: 34$ women) were recruited through advertisements on the radio, in print, and on flyers. Individuals with AUD ( $\mathrm{N}=58$ : 14 women) with 21-90 days of abstinence were recruited from North Central Florida treatment facilities for alcohol and substance use. Participants completed paper-and-pencil assessments addressing demographics, current mood [Beck Depression Inventory (BDI; Beck, Steer, \& Brown, 1996), Spielberger State Anxiety Inventory (AI; Spielberger,1983)] and substance-use histories including chronicity, quantity, and frequency of alcohol use [Quantity Frequency Index and maximum consumption in the previous 6 months Max-Quantity, Cahalan et al., 1969]. Medical histories were obtained by self-report. Individuals who reported significant neurologic disorder/insult, medical conditions, or medication use known to compromise neurobehavioral function (e.g. history of stroke, untreated hypertension, benzodiazepines) were excluded from the study. Participants were aged 25-59 and had 10-16 years of education.

\section{Working Memory Task}

Participants completed a modified version of a WM directed attend/ignore task (Gazzaley et al., 2005). This task involves presentation of two classes of stimuli (i.e. faces and scenes) in which participants are instructed to remember faces and ignore scenes in one condition and to remember scenes and ignore faces in a second condition. Each trial consisted of a series of cue images of 2 emotional or neutral faces and 2 non emotional scenes (all grayscale) presented in a pseudorandom order. There are six possible orders of stimulus presentation (e.g. FFSS, SSFF, 
FSFS) and three facial expressions (i.e. fearful, neutral, happy). The facial expression was consistent within trial. Pseudorandomization was conducted within blocks of six trials to confirm equal distribution of presentation order across the entire task. Cue stimuli were presented for $800 \mathrm{~ms}$ followed by a $200 \mathrm{~ms}$ fixation ' + '. Following presentation of the cue stimuli, a ninesecond delay period represented by a fixation cross occurred. Participants were instructed to maintain the to-be- remembered class of stimuli. EEG was analyzed from this stage of the task for the purpose of this paper. Afterward, the probe image was presented for $1500 \mathrm{~ms}$ and participants were asked to indicate via button press with the index finger of their dominant hand whether the probe image was present in the preceding cue set. Probe images were consistent with the instructions given (i.e. Remember Faces probes were always faces). For the purpose of this study the focus will be on the Remember Faces condition. A third condition instructed participants to passively view cue stimuli and respond with a button press to the direction of an arrow at the end of each trial. This condition was used as a baseline covariate.
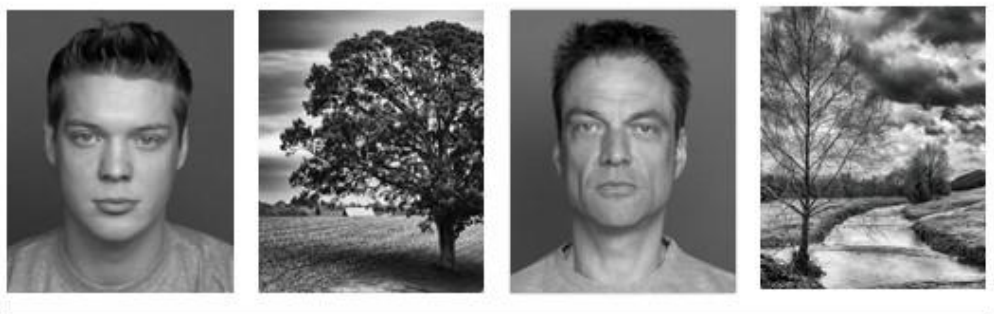

Cue Stimuli

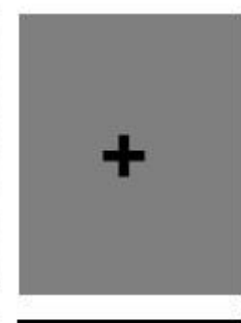

Delay

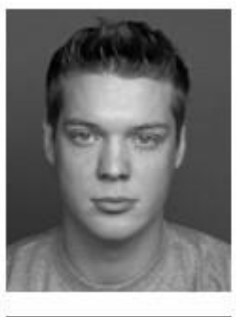

Probe

Figure 1. Working Memory Task: Remember Faces. In the trial depicted here participants were instructed to remember faces and ignore scenes. Cue stimuli were presented on a computer screen one at a time for $800 \mathrm{~ms}$ each (interstimulus interval $=200 \mathrm{~ms}$ ). Following presentation of all four stimuli was a $9000 \mathrm{~ms}$ delay (represented by a fixation cross). During this time participants were instructed to focus on the ' + ' and think about the previously presented face stimuli. The delay period was followed by the probe (1500ms) and an additional 2500ms response window. Participants were instructed to answer with a button press whether the probe matched one of the previously presented cue stimuli.

\section{EEG Recording}

Electrophysiological indices were collected throughout the task. Participants were fitted with a 64-channel array elastic cap arranged in an expanded international 10/20 System configuration. A mid-forehead ground was used, as well as linked electrodes attached to each earlobe as the reference. Two additional electrodes were placed above and below the outer canthus of the left eye to detect blinks and eye movements. Movement artifacts during data collection were minimized by participants' use of a chin rest attached to the top of the testing 
table. Impedances were maintained at or below 10kOhms. All procedures were conducted in a sound-attenuated, electrically shielded booth (Eckel Industries of Canada Limited, Morrisburg, $\mathrm{ON})$. The task was presented on a 17 -inch LCD monitor approximately $70 \mathrm{~cm}$ from participants. E-Prime software was utilized for task presentation. An additional computer using NeuroScan 4.4 Acquire software recorded continuous electroencephalography.

Data extracted from the nine second maintenance period were processed using the EEGLab toolbox in MatLab. Consistent with Boissoneault et al., 2016, 11 posterior electrodes were selected (PZ, P1, P2, P3, P4, PZP, P1P, P2P, OZ, O1, O2) to target PAP. A seven step automated data rejection process was utilized to identify and removed abnormal, improbable, and noisy data. Data were divided into 3-second epochs and filtered between .01 and $50 \mathrm{~Hz}$ to remove high and low frequency shifts. Eye-blinks and movement artifacts were removed using ADJUST. Three-second epochs containing excessive kurtosis, excessive moments and high frequency noise were deemed unusable. Following completion of data processing, useable data existed for 92 subjects (21 HC men, 29 HC women, 33 AUD men, 9 AUD women). Each individual's absolute power was averaged across the alpha frequency band (7-12 Hz).

\section{Statistical Analyses}

The statistical analyses were run in SAS version 9.4. The participants were compared by group and sex using ANOVA on basic demographic variables such as age, smoking status, and alcohol use in the past 6 months to ensure any preexisting differences would not confound the analyses of primary interest.

Next, a mixed linear model that compared PAP between group (HC and AUD) and sex (male and female), within emotion (i.e. fearful, neutral, happy) controlling for alpha power in the passive condition, was used. For significant between-subjects main and interactive effects, pairwise comparisons were analyzed within group and within sex. Statistics are reported with Fand p-values.

Lastly, behavioral performance reported in Lewis et al. (under review in revision), indicated no differences in accuracy between men and women with or without AUD. In light of equal performance between groups, a mixed linear model was used to explore the possible differential relationship between group's PAP during the 9 second delay period and previously reported WM task performance. The outcome variable was percent accuracy on the task with group, sex, and PAP as predictors. 


\section{Results}

\section{Demographics}

Demographics are shown in Table 1. The mean age of the $\mathrm{HC}$ was $43.3(\mathrm{SD}=12.5)$, and the mean age of individuals with AUD was $40.4(\mathrm{SD}=8.9)$. As previously reported in the treatmentseeking population (Lewis et al., 2019; Lewis et al., 2020; Hoffman, Lewis, \& Nixon, 2019), the groups significantly differed on typical alcohol consumption, binge alcohol consumption, BDI, AI and smoking habits (all p's<.05). There were no significant differences between men and women within each group.

Table 1. Demographics

\begin{tabular}{lllll}
\hline & HC Men & HC Women & AUD Men & AUD Women \\
\hline $\mathrm{N}$ & 27 & 34 & 44 & 14 \\
Age & $41.5(12.1)$ & $45.0(12.8)$ & $41.8(8.3)$ & $39.0(9.5)$ \\
\% Caucasian & 70.4 & 70.6 & 72.7 & 85.7 \\
Education & $14.6(1.5)$ & $14.9(1.5)$ & $13.2(1.6)$ & $13.0(1.6)$ \\
\% Smoker* & 17.9 & 6.3 & 64.3 & 85.7 \\
Average Drinks/Day** & $0.7(0.9)$ & $.35(.30)$ & $27.09(16.49)$ & $23.41(20.61)$ \\
BDI* & $3.8(4.3)$ & $5.6(6.7)$ & $14.2(9.2)$ & $15.6(10.0)$ \\
AI* & $43.5(7.1)$ & $42.9(7.6)$ & $56.0(13.2)$ & $58.5(15.9)$ \\
Yrs of Alcohol Problems & - & - & $18.5(8.5)$ & $15.3(10.3)$ \\
\hline
\end{tabular}

Note. A As measured by the Quantity Frequency Index (Cahalan, 1969)

B Beck Depressive Inventory (Beck, 1979)

C Spielberger State Trait Anxiety Inventory (Spielberger, 1983)

$*$ AUD $\neq$ HC, $\mathrm{p}<.05$

\section{Delay Period Posterior Alpha Power: Passive Condition}

In the passive condition, when participants were not instructed to focus either internally or externally, there was no significant difference between group or sex or across emotion in PAP (all p's > .59).

\section{Maintenance Period Posterior Alpha Power: Remember Faces}

Group differences are shown in Figure 2. A significant main effect of group was present $\mathrm{F}(1$, $87)=4.55, \mathrm{p}=.04$. Individuals with AUD had lower PAP compared to that of $\mathrm{HC}\left[\mathrm{M}_{\mathrm{HC}}=5.31\right.$ $(0.12)$ vs. $\mathrm{M}_{\mathrm{AUD}}=4.77(0.17)$. A main effect of sex was present $\mathrm{F}(1,87)=9.08, \mathrm{p}=.003$. Women 
displayed lower PAP compared to men $\left(\mathrm{M}_{\mathrm{Men}}=5.36(0.11)\right.$ vs. $\mathrm{M}_{\mathrm{Women}}=4.73(0.17)$. There was no group-by-sex interaction nor were there main or interactive effects of emotion (p's >.30).

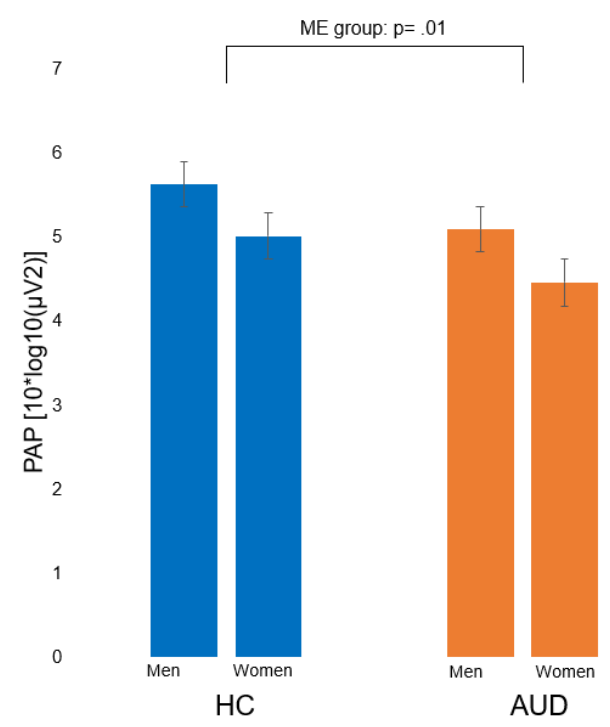

Figure 2. Posterior alpha power during WM Maintenance. Baseline-corrected least square means and standard error are depicted. A main effect of group was present ( $\mathrm{p}=.01$ ). Individuals with AUD had lower PAP compared to that of healthy controls. Though not hypothesized, a main effect of sex was also present ( $\mathrm{p}=.003$ ). Women displayed lower PAP compared to men. There was no interaction between group and sex.

\section{Relationship Between Posterior Alpha Power and Task Performance}

There was no main effect between PAP and task performance. An interaction of group and PAP on task performance was observed $\mathrm{F}(1,155)=11.66, \mathrm{p}<.001$, suggesting that PAP was differentially related to performance on the WM task between groups. PAP was not significantly related to accuracy for controls $(\mathrm{p}=.57)$. Among individuals with AUD, PAP was inversely related to performance (Figure 3 ). That is, lower PAP was associated with higher accuracy F(1, $37)=4.21, r^{2}=.10, p=.05$. This relationship did not differ between men and women $(p=0.16)$. 


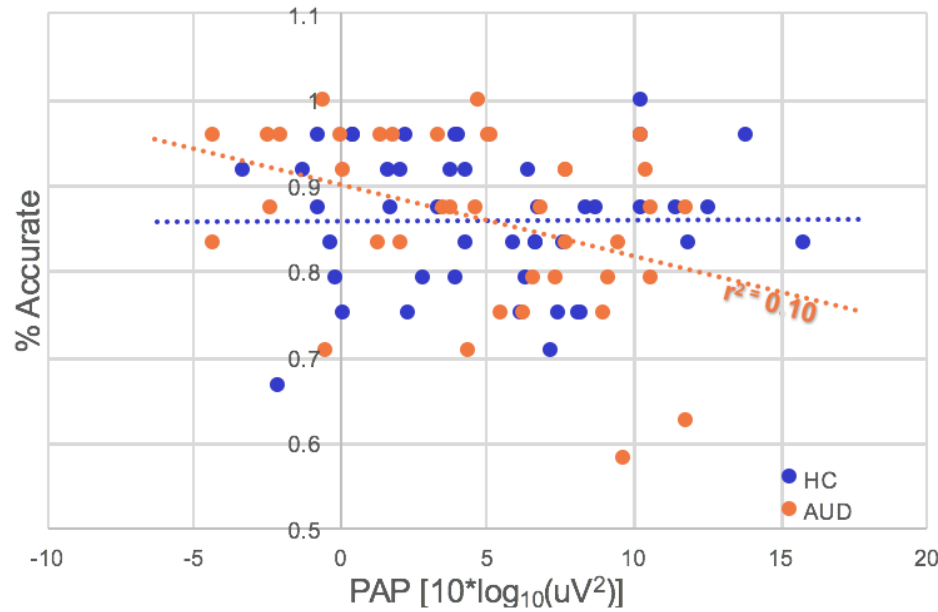

Figure 3. PAP was differentially related to performance on the WM task $(\mathrm{p}<.001)$. PAP was not related to accuracy for controls $(\mathrm{p}=.57)$. For individuals with AUD, lower PAP was associated with higher accuracy $\left(\mathrm{r}^{2}=.10\right.$, $\mathrm{p}=.05)$

\section{Discussion}

\section{Findings}

Decrements in the ability of individuals with AUD to perform well on WM tasks have been identified yet the underlying processes are not well understood. This project investigated differences in alpha power for individuals with AUD compared to $\mathrm{HC}$ during the maintenance phase of a WM task and how this relates to subsequent performance. Lower alpha power was present in individuals with AUD compared to that of HC. Additionally, there was an inverse relationship between performance and alpha power that was only present among individuals with AUD. This pattern has been seen in acute alcohol administration and aging and could be consistent with compensatory strategies (Boissoneault et al., 2016).

One possible strategy used by the AUD group could be enhanced external focus on the fixation cross to block out potential distractors (both external and internal). The heightened focus could manifest as increased PAP. As this task is relatively simple, the HC may not utilize the fixation cross as a means of protecting the stimuli. Another possible compensatory strategy could be the presence of other brain wave frequencies that were not assessed in the current work. That is, perhaps individuals with AUD are using different neural resources (e.g. frontal theta) during the task, however the current data don't speak to this possible function. Lastly, individuals with AUD show instability across multiple brain wave frequencies (Porjesz \& Begleiter 2003).

Examination of alpha waves relative to other frequencies (i.e. relative alpha power) may clarify this relationship. 


\section{Limitations}

Recruitment of women with AUD was challenging partially due to the limited number of women in treatment facilities, resulting in cell sizes that were not consistent across groups and restricted analyses of interaction within sex.

In addition to this, many participants were excluded from the study due to unusable EEG data effected by noise and various discrepancies in the collection process that did not allow us to properly extract their EEG data (e.g. high frequency shifts due to movement). While the use of a chinrest during EEG data collection aided in decreasing movement, some data loss still occurred.

\section{Conclusion}

Individuals with AUD showed lower PAP compared to HC during a WM maintenance task. Interestingly, there was an interaction with PAP and group on performance. Alpha power was inversely related to accuracy for individuals with AUD, although there was no relationship seen for the HC. Whether these findings are indicative of different behavioral or neural strategies is not clear and remains a question of interest. While the neurophysiological indices of other WM phases are well studied in AUD, this work provides greater clarity into neural alterations that occur during WM maintenance. Continued research into how neurological indices are altered in the absence of performance deficits in individuals with AUD is necessary.

\section{References}

American Psychiatric Association. (2013). Diagnostic and statistical manual of mental disorders (5th ed.). Arlington, VA: Author.

Baddeley, A. (1998). Working memory. Comptes Rendus de l'Académie des Sciences-Series III-Sciences de la Vie, 321(2-3), 167-173.

Baddeley, A. (2003). Working memory: looking back and looking forward. Nature reviews neuroscience, 4(10), 829-839.

Beatty, W. W., Tivis, R., Stott, H. D., Nixon, S. J., \& Parsons, O. A. (2000). Neuropsychological deficits in sober alcoholics: influences of chronicity and recent alcohol consumption. Alcoholism: Clinical and Experimental Research, 24(2), 149-154.

Beck, A. T. S. R., Steer, R. A. B. G., \& Brown, G. (1996). Manual for the Beck depression inventory-II (BDI-II).

Bernardin, F., Maheut-Bosser, A., \& Paille, F. (2014). Cognitive impairments in alcohol dependent subjects. Frontiers in psychiatry, 5, 78. 
Chanraud, S., Martelli, C., Delain, F., Kostogianni, N., Douaud, G., Aubin, H. J., ... \& Martinot, J. L. (2007). Brain morphometry and cognitive performance in detoxified alcohol-dependents with preserved psychosocial functioning. Neuropsychopharmacology, 32(2), 429-438.

Chanraud, S., Pitel, A. L., Müller-Oehring, E. M., Pfefferbaum, A., \& Sullivan, E. V. (2012). Remapping the brain to compensate for impairment in recovering alcoholics. Cerebral cortex, 23(1), 97-104.

Cohen, M. X. (2017). Where does EEG come from and what does it mean? Trends in neurosciences, 40(4), 208-218.

Corbetta, M., \& Shulman, G. L. (2002). Control of goal-directed and stimulus-driven attention in the brain. Nature reviews neuroscience, 3(3), 201-215.

De Bruin, E. A., Stam, C. J., Bijl, S., Verbaten, M. N., \& Kenemans, J. L. (2006). Moderate-to-heavy alcohol intake is associated with differences in synchronization of brain activity during rest and mental rehearsal. International Journal of Psychophysiology, 60(3), 304-314.

Gazzaley A., Cooney J.W., Rissman J., D’Esposito M. (2005). Top-down suppression deficit underlies working memory impairment in normal aging. Nature Neuroscience: Brief Report. Vol. 9(10). 1298-1300.

Geering, B. A., Achermann, P., Eggimann, F., \& Borbely, A. A. (1993). Period-amplitude analysis and power spectral analysis: a comparison based on all-night sleep EEG recordings. Journal of sleep research, 2(3), 121-129.

González-Garrido, A. A., López-Franco, A. L., Gómez-Velázquez, F. R., Ramos-Loyo, J., \& Sequeira, H. (2015). Emotional content of stimuli improves visuospatial working memory. Neuroscience Letters, 585, 43-47.

Gould, T. J. (2010). Addiction and cognition. Addiction science and clinical practice, 5(2), 4.

Hoffman, L. A., Lewis, B., \& Nixon, S. J. (2019). Neurophysiological and interpersonal correlates of emotional face processing in alcohol use disorder. Alcoholism: Clinical and Experimental Research, 43(9), 1928-1936.

Lee, H. J., \& Cho, Y. S. (2019). Memory facilitation for emotional faces: Visual working memory tradeoffs resulting from attentional preference for emotional facial expressions. Memory \& cognition, 47(6), 1231-1243.

Lewis, B., Boissoneault, J., Frazier, I., \& Nixon, S. J. (2016). Effects of age and acute moderate alcohol administration on neurophysiology during simulated driving. Alcoholism: Clinical and Experimental Research, 40(12), 2519-2527.

Lewis, B., Garcia, C. C., Bohan, R., \& Nixon, S. J. (2020). Impact of polysubstance use on social and non-affective cognitive performance among treatment-seeking individuals with alcohol use disorders. Addictive Behaviors, 106, 106359.

Lewis B., Price J.L., Garcia C., Ebner N.C., Nixon S.J., The impact of emotional face stimuli on working memory performance among men and women with alcohol use disorder. In revision at Addictive Behaviors. 
Lewis, B., Price, J. L., Garcia, C. C., \& Nixon, S. J. (2019). Emotional face processing among treatmentseeking individuals with alcohol use disorders: investigating sex differences and relationships with interpersonal functioning. Alcohol and Alcoholism, 54(4), 361-369.

Mather, M., \& Sutherland, M. R. (2011). Arousal-biased competition in perception and memory. Perspectives on psychological science, 6(2), 114-133.

Martin, E. (1968). Stimulus meaningfulness and paired-associate transfer: an encoding variability hypothesis. Psychological review, 75(5), 421.

Mueller-Oehring, E. M., Pfefferbaum, A., Sullivan, E. V., Colrain, I., \& Schulte, T. (2014). Brain Connectivity Changes Over Time In Alcoholism. Alcoholism: Clinical \& Experimental Research, 38,319 A.

Nixon, S. J., \& Glenn, S. W. (2002). Cognitive psychosocial performance and recovery in female alcoholics. In Recent developments in alcoholism (pp. 287-307). Springer, Boston, MA.

Oscar-Berman, M., \& Marinkovic, K. (2007). Alcohol: effects on neurobehavioral functions and the brain. Neuropsychology review, 17(3), 239-257.

Oscar-Berman, M., Valmas, M. M., Sawyer, K. S., Ruiz, S. M., Luhar, R. B., \& Gravitz, Z. R. (2014). Profiles of impaired, spared, and recovered neuropsychologic processes in alcoholism. In Handbook of clinical neurology (Vol. 125, pp. 183-210). Elsevier.

Porjesz, B., \& Begleiter, H. (2003). Alcoholism and human electrophysiology. Alcohol Research \& Health, 27(2), 153.

Seidel, E. M., Habel, U., Kirschner, M., Gur, R. C., \& Derntl, B. (2010). The impact of facial emotional expressions on behavioral tendencies in women and men. Journal of Experimental Psychology: Human Perception and Performance, 36(2), 500.

Stoica, P., \& Moses, R. L. (2005). Spectral analysis of signals. Upper Saddle River, NJ; Pearson/Prentice Hall. 